TUMOUR MICROENVIRONMENT

\section{Bacterial residents}

The presence of bacteria in human tumours was first recognized over 100 years ago, and yet our under-

the tumour microbiome may impact many aspects of tumour biology standing of this relationship has made little progress since owing to the challenges associated with profiling the low biomass of the intratumour microbiome. Nejman et al. have now overcome this barrier to comprehensively characterize the bacteria present in 1,526 human tumours and tively showed that LPS could be appeared to be mostly restricted to $16 \mathrm{~S}$ rRNA was also detected in all their adjacent normal tissues encompassing seven different solid tumour types. Their initial findings suggest that much like the gut microbiome, the tumour microbiome may impact many aspects of tumour biology.

To characterize the intratumour bacteria, the authors applied a set of varying approaches with multiple controls, which would enable them to detect bacterial DNA, RNA and outer membrane or cell wall constituents while eliminating potential contaminants. The seven tumour types included breast, ovarian, lung and pancreatic tumours as well as tumour types in which the composition of the microbiome has so far not been reported, such as melanoma, bone and brain tumours.

By first quantifying bacterial DNA, it was observed that bacteria were present in all of the different

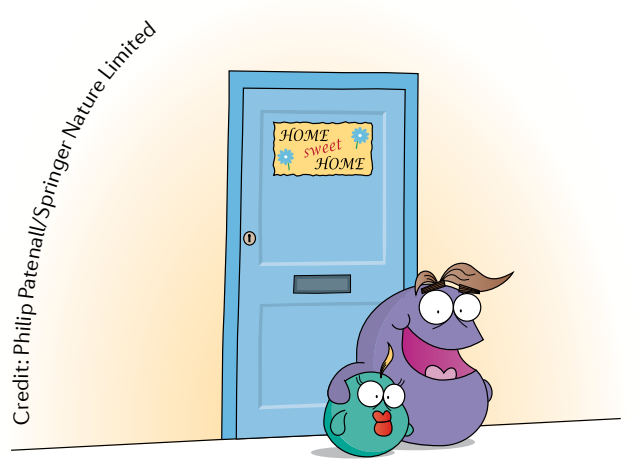

tumour types but to varying degrees. Next, immunohistochemical staining for bacterial LPS and lipoteichoic acid (LTA), which reveals Gram-negative and Gram-positive bacteria, respecfound in all tumour types while LTA melanomas. Furthermore, bacterial of the tumour types. Closer examination of the intratumour bacteria revealed that they were primarily intracellular, being localized within both cancer cells and immune cells. Interestingly, correlative light and electron microscopy (CLEM) demonstrated that the intracellular bacteria in breast tumours were perinuclear, although the reasoning for this positioning in the cell remains unknown. To exclude the possibility that the bacterial components present in tumours were not simply remnants, slices of freshly resected breast tumours were cultured ex vivo with fluorescently labelled D-alanine used in the generation of peptidoglycan, a key component of the bacterial cell wall. This revealed intracellular staining for D-alanine in breast tumours in line with the presence of live, metabolically active bacteria.

Next, the authors characterized the intratumour microbiome of each tumour type and their normal adjacent tissue (NAT). Surprisingly, this showed that breast tumours, which arise in what is typically considered an aseptic tissue, had a microbiome that was more diverse and richer than that of the other tumour types. Overall, the different tumour types each had a distinct microbial composition or signature, which was comparable to their NAT. For example, the most abundant bacterial phylum of pancreatic cancer was Proteobacteria akin to the normal duodenal microbiome, suggesting retrograde translocation of bacteria from the duodenum to the pancreas could account for this. Furthermore, there were bacterial species-specific differences between tumour types. Unexpectedly, Fusobacterium nucleatum, recognized to be prevalent in colorectal tumours, was also found to be abundant in breast and pancreatic tumours. Divergent microbial compositions were not only seen at the level of tumour type but also at the level of subtype. For example, between the $\mathrm{ER}^{+}, \mathrm{PR}^{+}$and HER2 ${ }^{+}$ subtypes of breast cancer, there were differences in the bacterial taxa that were most dominant.

Last, the authors chose to look at the microbial metabolic pathways of the different tumour types. This demonstrated that distinct microbial functions were associated with particular tumour types, indicating that increased levels of certain metabolites might be generating specialized niches for bacterial species who can utilize such metabolites. For instance, bacterial metabolic pathways associated with the degradation of chemicals in cigarette smoke, such as nicotine, were enriched in lung cancers of smokers compared with those who have never smoked. Notably, there were also differences in the bacterial taxa found in melanomas from patients who responded to immune checkpoint therapy relative to those who did not respond, potentially related to the observation that bacteria were also found in immune cells of tumours.

An exciting question for future research will be to ascertain whether these bacterial residents are in fact beneficial to the tumour or if their presence in human tumours is simply a mechanism enabling their own survival.

Anna Dart

ORIGINAL ARTICLE Nejman, D. et al. The human tumor microbiome is composed of tumor typespecific intracellular bacteria. Science $\mathbf{3 6 8}$, 973-980 (2020) 